\title{
Second harmonic generation in a fiberized amorphous silicon metamaterial
}

\author{
Jie Xu${ }^{1}$, Eric Plum ${ }^{1}$, Vassili Savinov ${ }^{1}$, and Nikolay I. Zheludev ${ }^{1,2}$ \\ 1. Optoelectronics Research Centre and Centre for Photonic Metamaterials, University of Southampton, Southampton, SO17 1BJ, UK \\ 2. Centre for Disruptive Photonic Technologies, SPMS, TPI, Nanyang Technological University, Singapore, 637371, Singapore \\ Authore-mail address: jx2n17@soton.ac.uk,erp@orc.soton.ac.uk,niz@orc.soton.ac.uk
}

\begin{abstract}
Patterning of amorphous silicon with chevron grooves yields a metamaterial frequency converter with a resonant second harmonic conversion efficiency of $\sim 10^{-11} / \mathrm{W}$, exceeding the previously achieved value for silicon metamaterial by two orders of magnitude. (C) 2020 The Author(s)
\end{abstract}

\section{Introduction}

Second-order nonlinear optical response provides an opportunity for control of light with light, which is essential if photonics should ever provide an answer to the exponential growth of the world's data processing requirements. Silicon-based photonics is driving the miniaturization and integration of optical circuits, but bulk silicon lacks second-order optical nonlinearity. We demonstrate second harmonic generation (SHG) by an amorphous silicon metamaterial. The nanostructure was fabricated on the tip of an optical fibre that collects and guides the generated light. The silicon metamaterial is a double chevron array that supports a closed-mode resonance with a quality factor of about 30 at a wavelength of $1510 \mathrm{~nm}$. At the resonance, the metamaterial's normalized second harmonic conversion efficiency is $\eta /\left(I L^{2}\right) \sim 10^{-11} / \mathrm{W}$, where $\eta$ is absolute conversion efficiency, $I$ is intensity and $L$ is interaction length. The observed normalized and absolute conversion efficiencies exceed earlier silicon metamaterial [1] by two and four orders of magnitude, respectively.
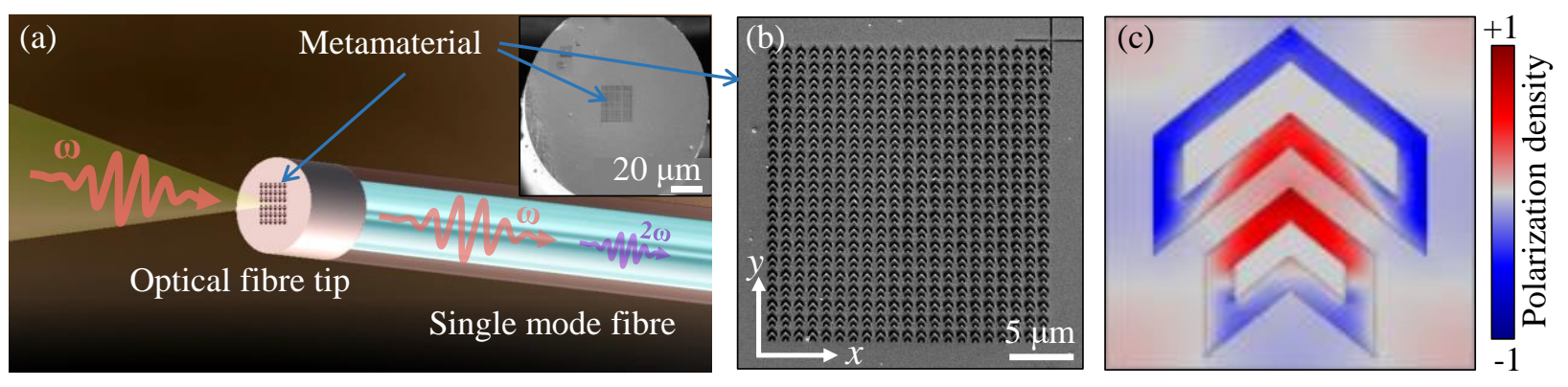

Fig. 1. Nonlinear silicon metamaterial on a fibre tip. (a) Artistic impression illustrating second harmonic generation by the resonant double chevron metamaterial. The inset SEM image shows the metamaterial on the core of a cleaved single mode optical fibre. (b) SEM image of the entire nonlinear metamaterial consisting of pairs of chevron grooves in silica coated with an amorphous silicon layer of $90 \mathrm{~nm}$ thickness. (c) The $y$ component of the linear polarization density at the metamaterial's closed-mode resonance excited by $y$-polarized light at $1510 \mathrm{~nm}$ wavelength.

\section{Results}

The silicon-on-silica metamaterial (Fig.1a,b) consists of an array of pairs of V-grooves of different size. Due to the different sizes of the grooves, the structure supports a closed-mode (Fano-type) resonance, characterized by an antisymmetric excitation (Fig. 1c). The resonant wavelength is $\lambda_{0}=1.5 \mu \mathrm{m}$ wavelength and its quality factor is 30 (Fig.2a). The metamaterial was fabricated by milling 256-nm-deep grooves into a cleaved silica fiber tip (covered entire fiber core, shown in Fig.1a,b) and then depositing a layer of 90-nm-thick CVD-silicon film on the structured fiber tip, resulting in an overall metamaterial thickness of $\mathrm{L}=346 \mathrm{~nm}$.

Second-harmonic response of the metamaterial was characterized by pumping the nanostructure with normally incident 200 fs laser pulses of $80 \mathrm{MHz}$ repetition rate and up to $9 \mathrm{GW} / \mathrm{cm}^{2}$ peak intensity, see Fig.1a. Strong second harmonic emission from the metamaterial is observed for $y$-polarized pump light at $1510 \mathrm{~nm}$ wavelength, Fig. 2a, 
while no second harmonic above the noise level is detected from unstructured reference samples (bare fibres with and without unstructured 90-nm-thick CVD-silicon film). A quadratic power dependence of the metamaterial's SHG signal is confirmed by Fig. $2 \mathrm{~b}$. The second harmonic spectral peak coincides with the metamaterial's absorption resonance, indicating that SHG is enhanced by the metamaterial's closed-mode resonance.

The second harmonic generation power conversion efficiency is $\eta=0.8 \times 10^{-10}$, which is comparable to efficiencies in metallic structures and exceeds previously reported silicon metamaterial by four orders of magnitude [1]. Considering that phase-matched SHG conversion efficiency increases linearly with pump peak intensity $I$ and quadratically with interaction length $L$, the corresponding normalized efficiency is $\eta /\left(I L^{2}\right)=8 \times 10^{-3} / \mathrm{GW}$, corresponding to $\chi_{y y y}{ }^{(2)} \sim 0.3 \mathrm{pm} / \mathrm{V}$, which is comparable to KDP. The normalized efficiency reported here exceeds previous silicon metamaterial [1] by two orders of magnitude. This improvement is largely due to resonant enhancement of second harmonic generation at the closed-mode resonance of the double chevron structure.

Three symmetry-allowed second-order susceptibility tensor components of the D1-symmetric metamaterial were extracted by fitting the pump-polarization-dependent intensity of generated second harmonic signal, giving $\left|\chi_{\mathrm{xyx}}{ }^{(2)} / \chi_{\mathrm{yyy}}{ }^{(2)}\right|=0.2$ and $\left|\chi_{\mathrm{yxx}}{ }^{(2)} / \chi_{\mathrm{yyy}}{ }^{(2)}\right|=0.2$ at $1510 \mathrm{~nm}$ pump wavelength. The fitted dependence of SHG on the orientation of linear pump polarization (Fig.2b inset) shows clearly that $\chi_{\mathrm{yyy}}{ }^{(2)}$ is the dominant susceptibility tensor component.

(a)

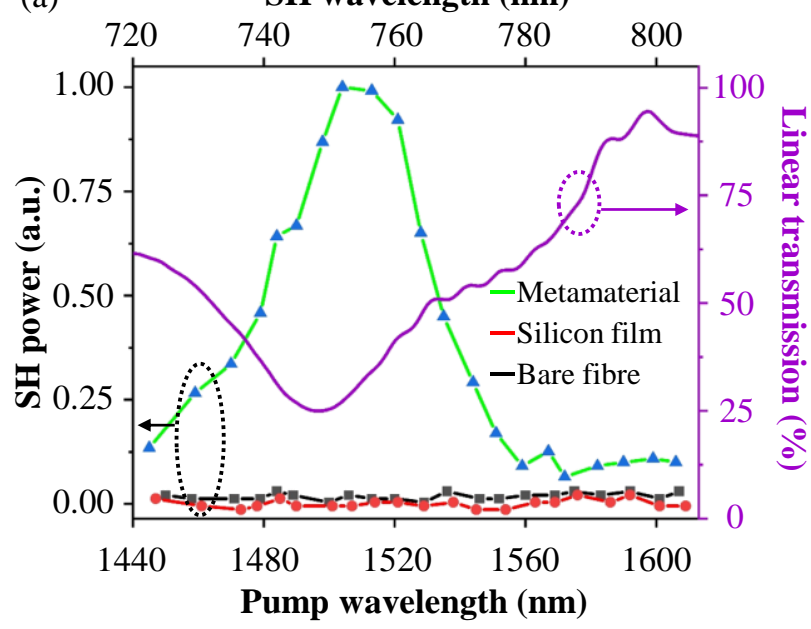

(b)

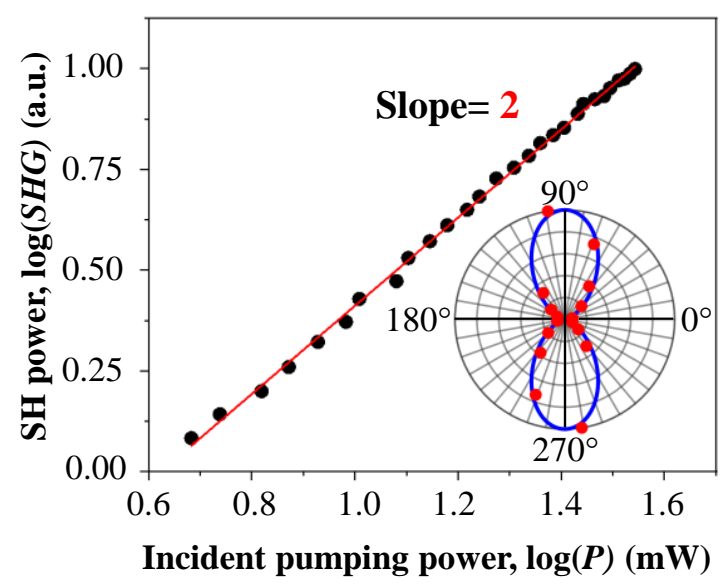

Fig. 2. Second harmonic generation by a nonlinear silicon metamaterial on a fibre tip. (a) Detected spectral dependence of second harmonic (SH) emission by metamaterial (green), unstructured silicon film (red) and unstructured bare fibre (black) on a cleaved fibre alongside the metamaterial's linear transmission spectrum (purple). (b) Power dependence of second harmonic generation of the metamaterial (black dots) with a quadratic fit (red line) at $1510 \mathrm{~nm}$ pump wavelength. Incident (pump) light is $y$-polarized in all cases. The inset shows generated second harmonic power as a function of pump polarization at $1510 \mathrm{~nm}$ pump wavelength (the resonance). Dots indicate measurements and the blue curve shows a fit.

\section{Summary}

We report a fiberized silicon-on-silica metamaterial that offers a four orders of magnitude improvement in power efficiency of second harmonic generation over previously reported results. The frequency converter is the first example of integration of all-dielectric second-order nonlinear metamaterial with fiber optics. These results will be of interest for nonlinear optics as well as scientists and engineers working with light, silicon photonics, fibre optics, nanotechnology and/or metamaterials. 